\title{
Choroidal Folds in Acute-Stage Vogt-Koyanagi-Harada Disease Patients with Relatively Short Axial Length
}

\author{
Minoru Tanigawa Haruyuki Ochiai Yoko Tsukahara \\ Yuko Ochiai Hiromitsu Yamanaka
}

Department of Ophthalmology, Shin-Nagata Eye Institute, Kobe City, Japan

\section{Key Words}

Vogt-Koyanagi-Harada disease - Choroidal folds · Shortened axial lengths · Acute stage

\begin{abstract}
Purpose: To report 2 cases of Vogt-Koyanagi-Harada disease accompanied by remarkable choroidal folds in the acute stage. The early indicator of recurrence in these 2 cases was the identification of choroidal folds by spectral-domain optical coherence tomography (SD-OCT). Case Reports: A 68-year-old woman (Case 1) presented with visual loss in both eyes. Funduscopic examination revealed optic disc swelling and serous retinal detachment in both eyes. SD-OCT revealed remarkable choroidal folds and serous retinal detachment. After the initiation of systemic steroid treatment, choroidal folds disappeared rapidly and the amount of serous retinal detachment reduced remarkably. Choroidal folds observed on SD-OCT were the early indicators of recurrence prior to the emergence of serous retinal detachment. $A$ 62-year-old woman (Case 2) presented with bilateral blurred vision and metamorphopsia. SD-OCT showed remarkable choroidal folds and serous retinal detachment in both eyes. After the initiation of systemic steroid treatment, choroidal folds and serous retinal detachment disappeared. At the time of recurrence, choroidal folds were observed by OCT. Discussion: During monitoring of Vogt-Koyanagi-Harada disease treatment, choroidal folds could be an early sign of recurrence. When choroidal folds are observed and recognized as an early indicator of recurrence, a prompt increase in steroids can improve the patients' prognosis. Finally, both cases presented here had relatively short axial lengths, and we speculate that a shortened axial length may be a cause of choroidal folds in the acute stage of the disease.
\end{abstract}




\section{Case Reports}

Case 1

A 68-year-old woman presented with visual loss in both eyes, which had persisted for 1 month. Best-corrected visual acuity (BCVA) measured using the Landolt ring chart was 0.4 in her right eye and 0.3 in her left eye. No remarkable findings or inflammation was observed in the anterior segment of either eye. Funduscopic examination revealed optic disc swelling and serous retinal detachment in both eyes (fig. 1a, b). Fluorescein angiography (FA) revealed remarkable leakage of the dye from the optic disc and retina in both eyes in the early phase, and subretinal dye pooling in both eyes was observed in the late phase (fig. 1c, d). Spectral-domain optical coherence tomography (SD-OCT; Cirrus ${ }^{\circledR}$ HD OCT; Carl Zeiss Meditec, Tokyo, Japan) revealed remarkable choroidal folds and serous retinal detachment in both eyes (fig. 1e, f). The patient also complained of headache and tinnitus. Brain MRI showed no intracranial mass lesion. Vogt-Koyanagi-Harada (VKH) disease was diagnosed on the basis of the patient's medical history as well as of her FA and SD-OCT findings. Steroid pulse therapy consisting of 1,000 mg methylprednisolone was intravenously administered for 3 days, followed by oral steroid tapering (fig. 2). After the initiation of systemic steroid treatment, SD-OCT showed that choroidal folds disappeared rapidly and the amount of serous retinal detachment reduced remarkably. BCVA improved to 1.0 in the right eye and 0.9 in the left eye before disease recurrence, which was confirmed by an OCT image showing serous retinal detachment (fig. $3 \mathrm{c})$. OCT detected choroidal folds 2 months before the emergence of serous retinal detachment (fig. $3 \mathrm{~b}$ ). At the time of recurrence, BCVA had not deteriorated and the patient did not complain of any visual disturbances. Steroid pulse therapy consisting of 1,000 mg methylprednisolone was initiated again for 3 days, followed by oral steroid tapering. After the re-introduction of steroid pulse therapy, SD-OCT showed disappearance of the choroidal folds. The patient is now stabilized without medication and observed periodically. Finally, axial lengths of her eyes were relatively short, $21.84 \mathrm{~mm}$ in the right eye and $21.87 \mathrm{~mm}$ in the left eye, as determined by A-mode echo measurement during disease remission.

\section{Case 2}

A 62-year-old woman presented with bilateral blurred vision that had persisted for 1 week and metamorphopsia that had emerged the day before. On her first visit, the patient's BCVA was 0.8 in her right eye and 0.5 in her left eye. She also suffered from headaches and tinnitus for 1 week.

Funduscopic examination revealed serous retinal detachment in both eyes without optic disc swelling (fig. 4a, b). SD-OCT showed remarkable choroidal folds and mutilobular serous retinal detachment in both eyes with the presence of subretinal septa (fig. 4c, d). After VKH diagnosis, systemic steroid therapy consisting of $250 \mathrm{mg}$ methylprednisolone was initiated and tapered (fig. 5). After systemic steroid therapy initiation, SD-OCT showed that choroidal folds disappeared and the amount of serous retinal detachment remarkably reduced in both eyes. BCVA improved to 0.9 in the right eye and 0.9 in the left eye before disease recurrence. At the time of recurrence, choroidal folds were detected by OCT and funduscopic examination, and the patient again started complaining of metamorphopsia (fig. 4e, f). The amount of methylprednisolone was increased to $40 \mathrm{mg}$ per day and was tapered again. After the increase in steroids administered, the patient recovered from metamorphopsia, and SD-OCT showed disappearance of the choroidal folds. The patient is now stabilized with $5 \mathrm{mg}$ methylprednisolone and observed periodically. Axial lengths of her eyes were relatively short, 22.49 $\mathrm{mm}$ in the right eye and $22.63 \mathrm{~mm}$ in the left eye, as determined by A-mode echo measurement during disease remission.

\section{Discussion}

Serous retinal detachment and subretinal septa are commonly observed on OCT in the acute stage of VKH disease, and FA shows leakage of the dye from the retina in the early phase and subretinal dye pool in the late phase [1-3]. Choroidal folds identified by SD-OCT are a recently reported finding in the acute stage of VKH disease $[4,5]$. In addition, Maruko et al. [6] reported that, in the acute stage, VKH disease cases presented with a thickening of the choroid. The underlying mechanism of choroidal fold formation remains unknown. 
In a previous report, choroidal folds were observed in $12 \%$ of VKH disease patients in the acute stage [4]. As shown in our 2 cases, choroidal fold identification by SD-OCT was useful as an early indicator of VKH disease recurrence during the course of treatment. SD-OCT images of Case 1 showed that localized serous retinal detachment emerged 2 months after the choroidal folds were detected again by SD-OCT after remission.

To date, intractable cases of VKH disease have proven to be problematic for the attending physicians. We speculate that among the intractable cases of VKH disease, there are recurring cases that have remained untreated for a long time, which was sufficient to destroy the retinal pigment epithelium and sensory retinal function because of the difficulty in detecting disease recurrence. For example, patients might have only slight choroidal folds or serous retinal detachment without remarkable visual acuity deterioration. However, SD-OCT allows physicians to identify disease recurrence earlier and re-start steroid therapy sooner, probably resulting in better prognosis as shown in Case 2.

It is also interesting to note that in both cases presented here, the patients had relatively short axial lengths and both experienced disease recurrence with choroidal folds as a preceding sign of acute inflammation. Wu et al. [4] hypothesized that marked congestion and thickness of choroids cause folding in order to adapt the unchanged intraocular volume. We speculate that limited ocular globe volume and fulminant inflammation could be factors causing choroidal folds in the acute stage. In limited spaces, such as in the relatively small ocular globe, acute inflammation causes thickening of the choroid adjacent to the solid sclera. There is a possibility that thickened choroidal structures are confined in the relatively small ocular globes and become voluminous and form choroidal folds in the acute stage of the disease. We also speculate that extraordinary thickening of the choroids caused by fulminant inflammation may cause choroidal folds in ordinary-sized ocular globes.

It is unknown whether eyes with choroidal folds or shortened axial lengths are more likely to suffer from VKH disease recurrence. Our experience using SD-OCT in VKH disease cases is limited, and more cases are needed to verify our hypothesis that eyes with shortened axial lengths may tend to have choroidal folds and thus may be more likely to experience VKH disease recurrence. Recently, enhanced depth imaging OCT has been reported to allow evaluation of choroidal thickness [6]. Considering such technological advances, more detailed analyses of the ocular globe structure in the acute stage of VKH disease are expected in the future. 


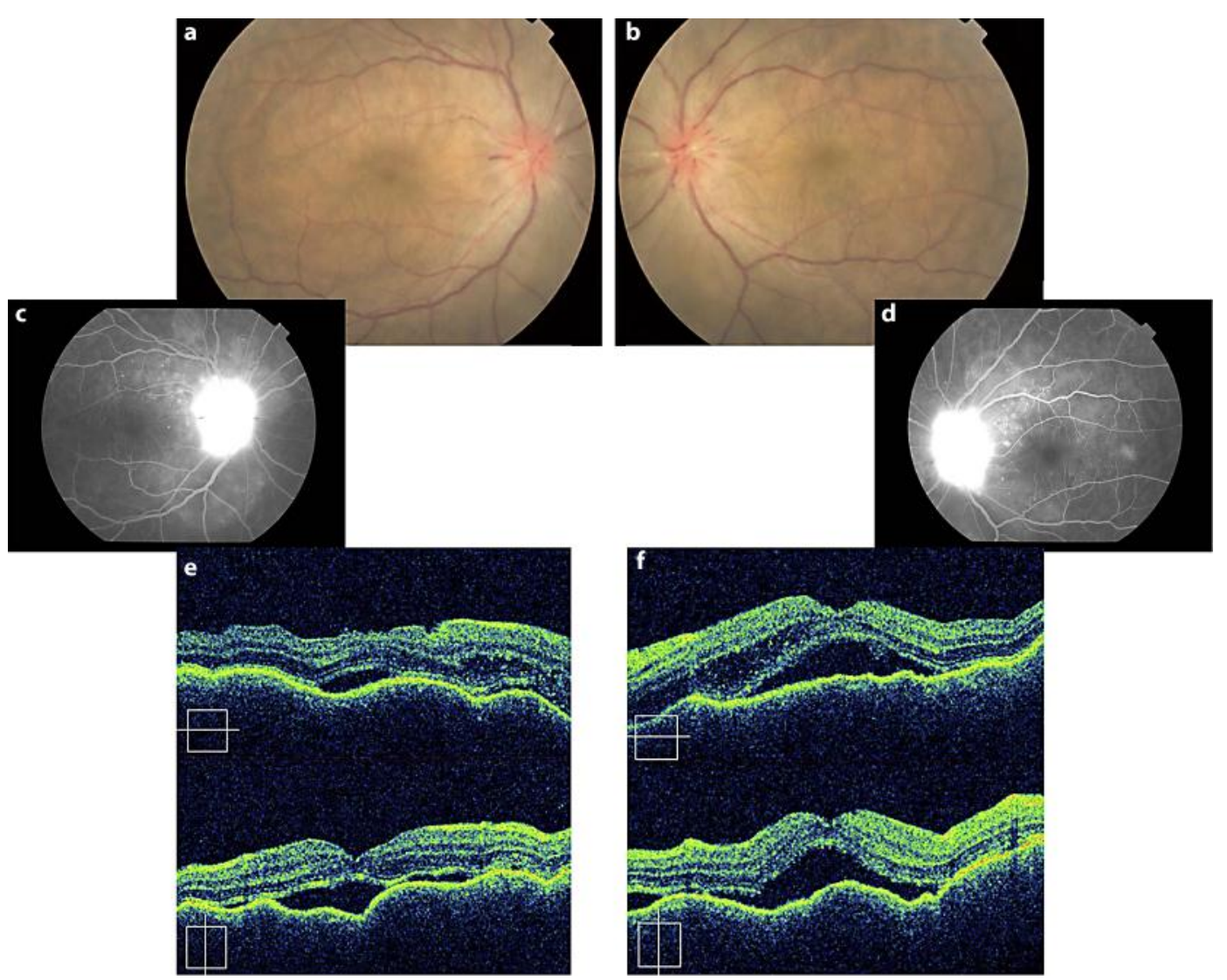

Fig. 1. a, b Fundus photography of optic disc swelling and serous retinal detachment in both eyes of Case 1 before treatment. c, d FA showing remarkable dye staining of the optic disc and subretinal dye pooling in both eyes in the late phase. e, $\mathbf{f}$ SD-OCT showing remarkable choroidal folds and serous retinal detachment in both eyes. 
Doses (mg) of methylprednisolone

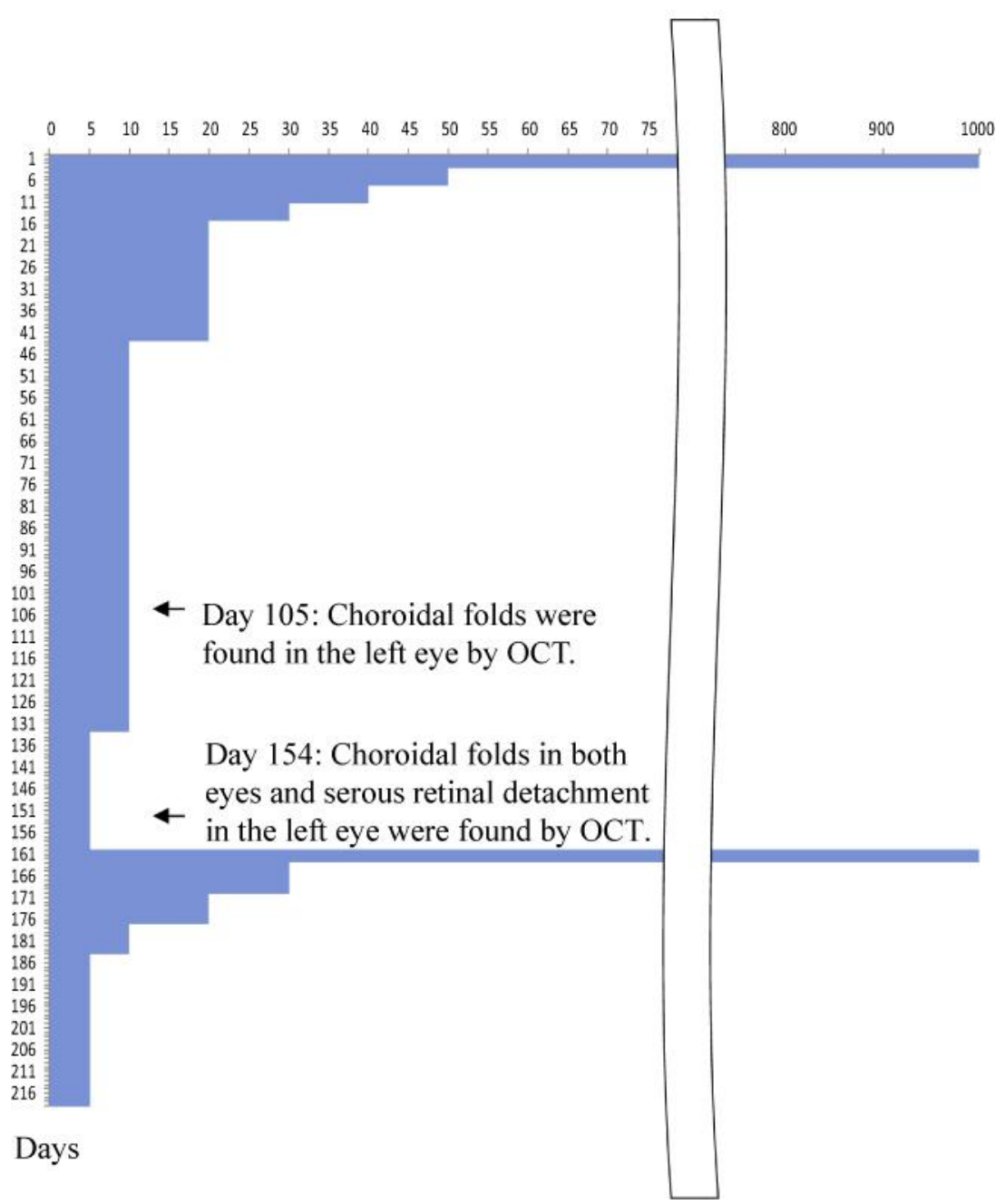

Fig. 2. Doses of methylprednisolone and treatment course of Case 1. Day 1 indicates the first day on which steroids were administered. On day 105, choroidal folds were detected by OCT. On day 154, serous retinal detachment was detected by OCT. Steroid pulse therapy was initiated again. 


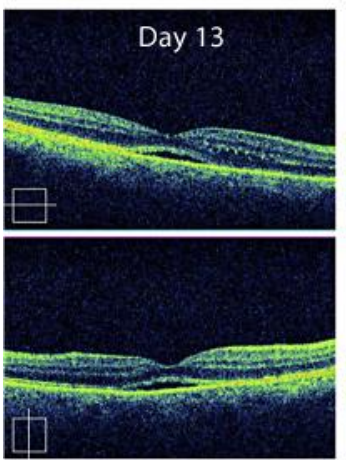

Right

c
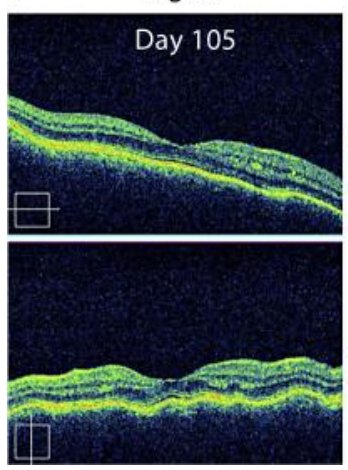

b
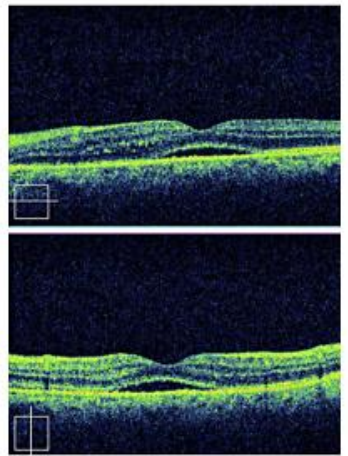

Left

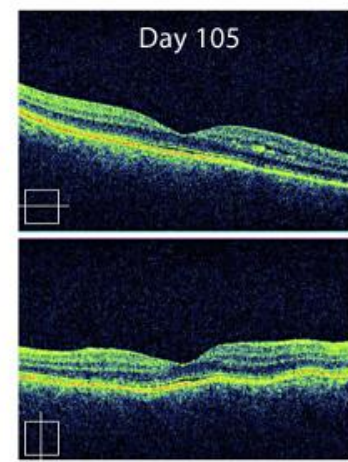

Right

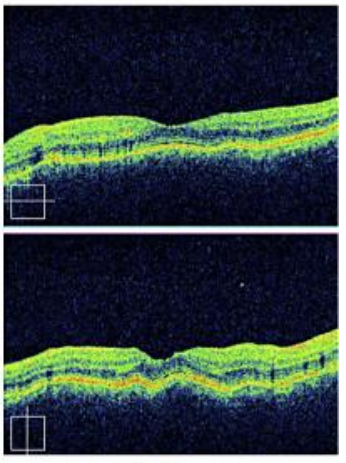

Left
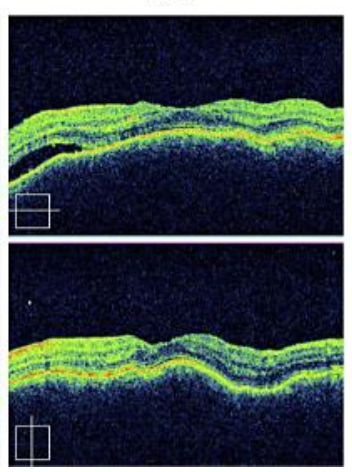

Fig. 3. OCT images of Case 1. a Choroidal folds rapidly disappeared and the amount of serous retinal detachment was reduced remarkably on day 13 after the initiation of steroid therapy. $\mathbf{b}$ Choroidal folds re-formed on day 105. c Serous retinal detachment appeared in the left eye and choroidal folds were enhanced on day 154. 


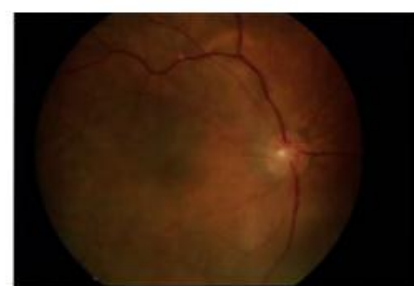

c
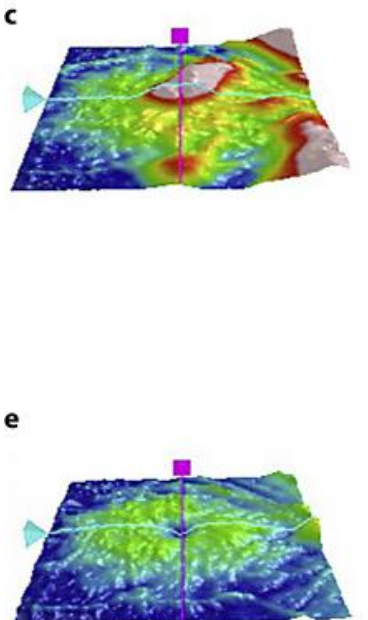
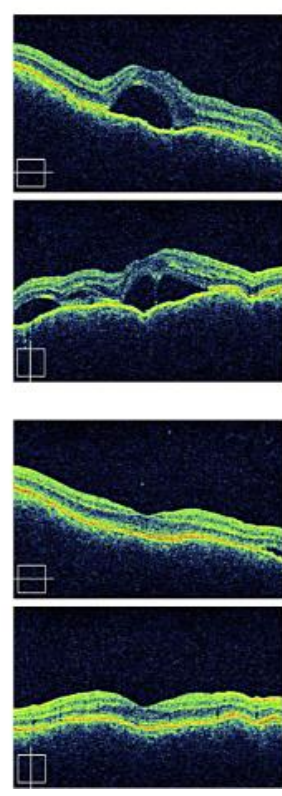

b
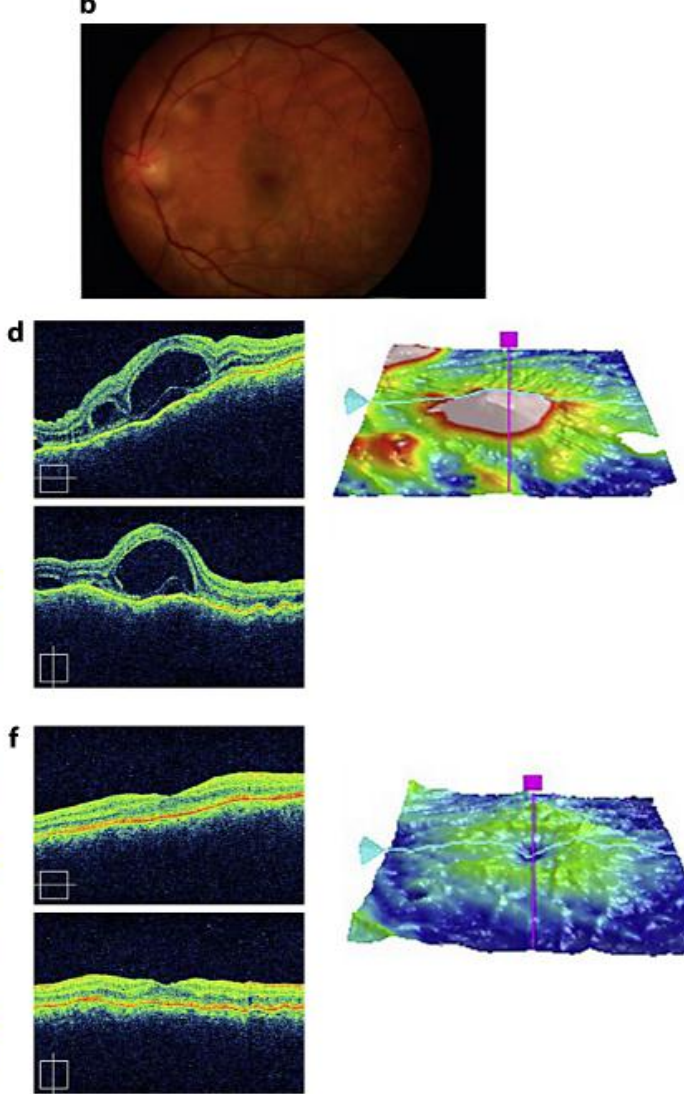

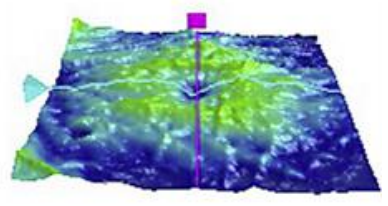

Fig. 4. a, $\mathbf{b}$ Fundus photography showing serous retinal detachment in both eyes of Case 2 before treatment. c, $\mathbf{d}$ SD-OCT showing choroidal folds and serous retinal detachment with the presence of subretinal septa before treatment. e, f SD-OCT images showing choroidal fold formation on day 63 after the initiation of steroid therapy. 


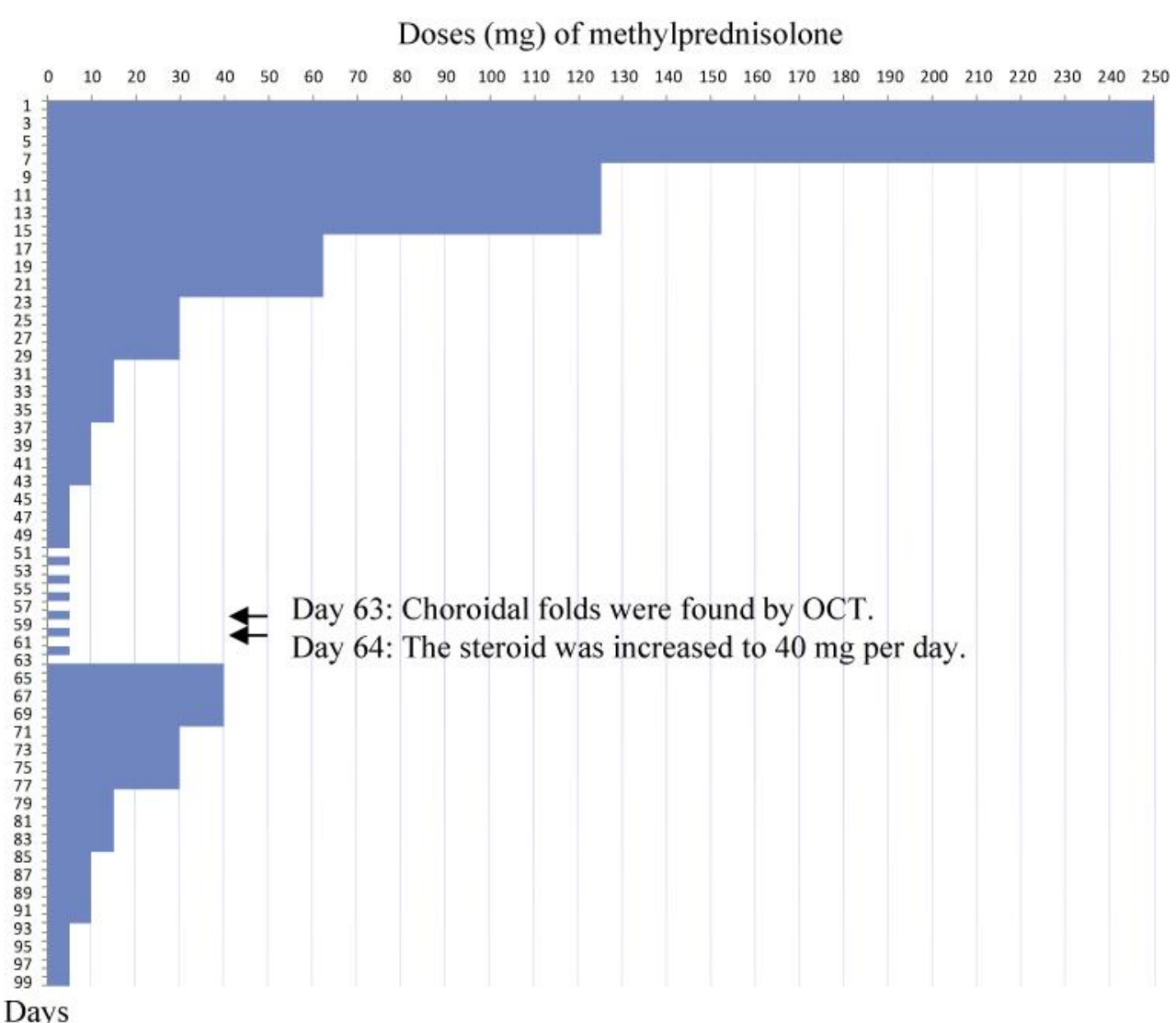

Fig. 5. Doses of methylprednisolone and treatment course of Case 2. Day 1 indicates the first day on which steroids were administered. On day 63 , choroidal folds were detected by OCT and funduscopic examination. On day 64 , the steroid was increased to $40 \mathrm{mg} /$ day.

\section{References}

1 Maruyama Y, Kishi S: Tomographic features of serous retinal detachment in Vogt-Koyanagi-Harada syndrome. Ophthalmic Surg Lasers Imaging 2004;35:239-242.

-2 Yamaguchi Y, Otani T, Kishi S: Tomographic features of serous retinal detachment with multilobular dye pooling in acute Vogt-Koyanagi-Harada disease. Am J Ophthalmol 2007;144:260-265.

-3 Tsujikawa A, Yamashiro K, Yamamoto K, Nonaka A, Fujihara M, Kurimoto Y: Retinal cystoid spaces in acute Vogt-Koyanagi-Harada syndrome. Am J Ophthalmol 2005;139:670-677.

4 Wu W, Wen F, Huang S, Luo G, Wu D: Choroidal folds in Vogt-Koyanagi-Harada disease. Am J Ophthalmol 2007;143:900-901.

5 Gupta V, Gupta A, Gupta P, Sharma A: Spectral-domain cirrus optical coherence tomography of choroidal striations seen in the acute stage of Vogt-Koyanagi-Harada disease. Am J Ophthalmol 2009;147:148153.

-6 Maruko I, Iida T, Sugano Y, Oyamada H, Sekiryu T, Fujiwara T, Spaide RF: Subfoveal choroidal thickness following treatment of Vogt-Koyanagi-Harada disease. Retina 2011;31:510-517. 